\title{
Nonretinotopic processing is related to postdictive size modulation in apparent motion
}

\author{
Takahiro Kawabe
}

Published online: 7 April 2011

(C) Psychonomic Society, Inc. 2011

\begin{abstract}
The present study briefly examined how the perceived size of a leading flash would be modulated by trailing motion signals. Observers were presented with two vertical green bars that were followed by white bars with different lengths, which were presented at different locations from the green bars. The task of observers was to discriminate which of the green bars was shorter than the other (Experiment 1) or whether the lengths of the green bars were equal or not (Experiments 2 and 3). One green bar producing apparent motion with the following shorter white bar was reported to be shorter than the other green bar producing apparent motion with the following longer white bar, not only when motion correspondence was determined on the basis of retinal proximity (Experiments 1 and 2),but also when motion correspondence was determined on the basis of nonretinotopic informationthat is, a relative location within each perceptual group of bars (Experiment 3). These results indicate that motion processing involving object updating or motion deblurring in the nonretinotopic frame of reference is related to postdictive size modulation.
\end{abstract}

Keywords Postdiction · Size $\cdot$ Apparent motion · Motion correspondence $\cdot$ Object updating

The visual system suffers from severe neural-processing delays in causing visual awareness from sensory inputs. Thus, the system tries to account for the neural-processing delays on the basis of either or both of the following two strategies. One strategy for compensatingfor the neural

\section{T. Kawabe $(\square)$}

Institute for Advanced Study, Kyushu University,

6-19-1, Hakozaki, Higashi ward,

Fukuoka 8128581, Japan

e-mail: takkawabe@gmail.com delays is to utilize currently acquired information to anticipate future events. This strategy of the visual system is called prediction, or,in motion perception specifically, extrapolation (Nijhawan, 2002, 2008). Motion extrapolation (or mental extrapolation) has been applied in the explanation for flash-lag effects (Nijhawan, 1994, 2002, 2008; Shi \& Nijhawan, 2008) and for forward displacements of the positions at which moving targets disappear (Freyd, 1987; Kerzel, 2003; Maus \& Nijhawan, 2006, 2008). That is, by predicting future events by utilizing acquired information, the visual system tries to fill temporal gaps between physical and mental events.

Another strategy for compensatingfor the neural delays is postdiction. It is assumed that the visual system continues to gather information within a temporal window even after the offset of the stimulus of interest and produces the final percept of the stimulus by integrating the stimulus representation with the later-occurred relevant events (Choi \& Scholl, 2006; Eagleman \& Sejnowski, 2007). Apparent motion is a representative phenomenon induced by postdiction. Specifically, motion direction of apparent motion is determined after the subsequent frames have been presented. Interestingly, the appearance of the motion trajectory of a two-stroke apparent motion is also postdictively modulated. In a two-stoke apparent motion consisting of flashes of a triangle and a square, observers report a flash whose shape gradually changing from a triangle to a square in motion trajectory (Kolers \& von Grünau, 1976). Intriguingly, for a motion trajectory of a two-stroke apparent motion consisting of red and green flashes, observers report an abrupt change of flash color from red to green at the middle of the motion trajectory (Kolers \& von Grünau, 1975). The data indicate that the appearance of apparent motion is strongly modulated by the following information. 
In addition to the appearance of motion trajectory between flashes, the appearance of the flash presented in the first frame is also modulated by the subsequent frame in a twostroke apparent motion. Eagleman and Sejnowski (2007) demonstrated that the initial location of a flash in the first frame was shifted toward the location of a flash in the second frame. Illusory displacement of the location at which a moving target appears in the direction of the subsequent motion has been called the Fröhlich effect" (Fröhlich, 1923). It has been considered that the Fröhlich effect stems from temporal interaction of metacontrast masking and attention shifts (Carbone \& Ansorge, 2008; Kirschfeld \& Kammer, 1999). However, the phenomenon reported by Eagleman and Sejnowski is not consistent with this conventional explanation for the Fröhlich effect, because if it stemmed from metacontrast masking, it would have resulted only in the reduction of visibility of the first frame, leading to no illusory displacements. Therefore, the illusory offset of a flash in the first frame toward a flash in the second frame requires explanations different from those for the Fröhlich effect.

In addition, Eagleman and Sejnowski (2007) showed that the apparent size of a moving object was modulated by successive size changes of following objects. Specifically, a colored flash in a contracting apparent motion comprising uncolored flashes was perceived to be smaller than the flash in an expanding apparent motion. This phenomenon was first reported by Cai and Schlag (2001) as "asynchronous feature binding," which assumes that a colored flash is perceptually bound with the following uncolored flash (i.e., for a contracting motion trajectory, a colored flash is perceptually bound with one of the smaller uncolored flashes), leading to size distortion. On the other hand, Eagleman and Sejnowski argued against this explanation, providing data showing that even when size distortion of a colored flash occurred depending on the size of the following uncolored flashes, the perceived number of uncolored flashes after the colored flash was veridical, suggesting that asynchronous feature binding was not suitable as an explanation of size distortion of the first frame in a two-stroke apparent motion (however, it is also possible that a colored flash is bound with an interpolated apparent motion trajectory, leading to a size distortion of the flash along a contracting/expanding apparent motion trajectory). These results indicate that the postdictive motion signals (i.e., motion signals after presentation of the critical flash to be assessed) may postdictively bias the localization of edges of the first flash, leading to size distortion. This type of mislocalization of static edges by motion signals has been reported repeatedly elsewhere (Shim \& Cavanagh, 2004, 2006; Whitney \& Cavanagh, 2000). Motion-induced mislocalization of edges may underlie size distortion as reported by Eagleman and Sejnowski.
The purposes of the present study were to (1) extend the finding of Eagleman and Sejnowski (2007) and (2) demonstrate a new aspect of the size modulation by postdiction.

For the first purpose, it is unclear whether the size distortion of a flash by the following flashes stems solely from postdictive motion signals after the first flashes, because few previous studies have examined the size modulation of a flash in the absence of leading sequences of flashes before the critical flash, the size of which was judged. Although Carbone and Ansorge (2008) examined the Fröhlich effect in size perception, they presented a smooth motion sequence after the initial frame, and hence, it was unclear whether their phenomenon stemmed from the modulation of visual size by postdictive motion signals or temporal interaction of metacontrast masking and attention shift. To disentangle these problems, the present study examined the size modulation by employing a two-stroke apparent motion, similar to the one in Eagleman and Sejnowski (2007).

For the second purpose, it is an intriguing question whether size modulation by postdictive motion signals occurs even when motion correspondence between two frames is determined by nonretinotopic information. In this study, the nonretinotopic information refers to relative position information within a perceptual group. Figure 1a represents a schematic explanation of motion correspondence on the basis of retinotopic information (i.e., spatial proximity), where $\mathrm{A} 1$ and $\mathrm{B} 1$ in frame 1 , correspond to $\mathrm{A} 2$ and B2, respectively, in frame 2. Likewise, Fig. 1b shows a schematic representation of motion correspondence on the basis of nonretinotopic information (i.e., relative location within a perceptual group). Specifically, within each frame, two vertical bars are perceptually grouped, and A1 (left) and B1 (right) correspond to A2 (left) and B2 (right), respectively, depending on the relative location of each grouped bar. If the retinotopic information is applied to a

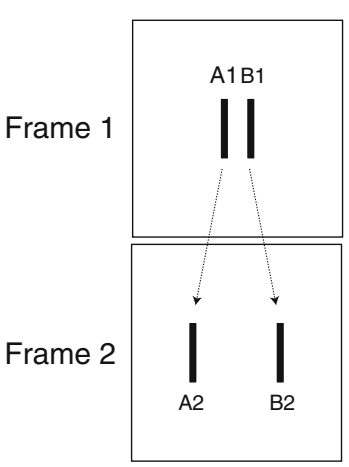

(a)

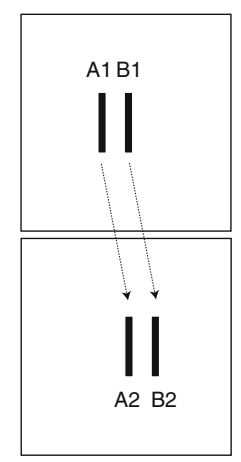

(b)
Fig. 1 Schematic explanations of motion correspondence on the basis of a retinotopic information and $\mathbf{b}$ nonretinotopic information. In both panels, A1 and B1 in frame 1 perceptually correspond to A2 and B2, respectively, in frame 2 
situation such as that shown in Fig. 1b, B1 will correspond to A2, although this type of percept does not occur.

Previous studies have shown that the visual system alters the appearance of moving objects on the basis of nonretinotopic information (Boi, Öğmen, Krummenacher, Otto, \& Herzog, 2009; Öğmen, Otto, \& Herzog, 2006; Otto, Öğmen, \& Herzog, 2008). Moreover, it has been shown that visual features within an object in the leading frame strongly modulate the appearance of visual features within an objet along a motion trajectory (Breitmeyer, Herzog, \& Öğmen, 2008; Kawabe, 2008). However, none of these studies showed that postdictive motion signals depending on nonretinotopic information could induce size distortion of a preceding flash. The second purpose of the present study was to address this issue.

Experiment 1 was performed to assess whether size modulation by postdictive motion signals would occur even in two-stroke apparent motion. However, because the task of observers was to judge which of the target bars was shorter (i.e., a comparative task), the observers in Experiment 1 might have based their response on the perceived size of the post bars that followed the target bars they were meant to judge. In Experiments 2, by using an equality judgment task in which the observers judged whether the sizes of the target bars were the same or different, it was confirmed that postdictive size modulation did not stem from a bias to respond to the length pattern of the post bars (see Experiments 2 for details of the rationale as to why we could remove the involvement of response bias). Finally, in Experiment 3, it was examined whether size modulation is induced by postdictive motion signals where motion correspondence was determined on the basis of nonretinotopic information. On the basis of the results, we discuss the role of higher-level motion processing relevant to object updating (Lleras \& Moore, 2003) or motion deblurring in postdictive size modulation.

\section{Experiment 1}

Method

\section{Observers}

Six observers (including the author) participated in this experiment. All subjects had normal or corrected-to-normal visual acuity and gave written informed consent before the experiment. With the exception of the author, they were unaware of the purpose of the study.

\section{Apparatus}

The stimuli were presented on a 19-in. CRT monitor (RDF193H, Mitsubishi) with a resolution of $1,024 \times 768$ pixels and a refresh rate of $100 \mathrm{~Hz}$. Presentation of stimuli and collection of data were controlled by a computer (Mac Pro, Apple). A chinrest (HE284, Handaya) was utilized to stabilize observers' visual field. The color and luminance of the stimuli were measured using a colorimeter (CL-100, Minolta). The experimental program was written using PsychToolBox 3 (Brainard, 1997; Pelli, 1997).

\section{Stimuli}

The stimuli consisted of a fixation dot, a background, two green target bars, and two white post bars (see Fig. 2a).

Fixation dot and background The size of the fixation dot was $0.15^{\circ} \times 0.15^{\circ}$, and its color was red (CIE [1931]; $x=$ $0.612, y=0.346, Y=23.7)$. The fixation dot was always presented at the center of the display. The luminance of the background was $32.7 \mathrm{~cd} / \mathrm{m}^{2}$.

Target bars The color of the target bars was green $(x=$ $0.283, y=0.588, Y=87.2)$. Each of the target bars was centered at $0.37^{\circ}$ left or right of and $2.22^{\circ}$ above the fixation dot. Their lengths were changed relative to one another on each trial; when the length of the left bar was $0.49^{\circ}, 0.77^{\circ}$, $0.91^{\circ}, 1.05^{\circ}, 1.19^{\circ}, 1.33^{\circ}$, or $1.61^{\circ}$, the length of the right bar was $1.61^{\circ}, 1.33^{\circ}, 1.19^{\circ}, 1.05^{\circ}, 0.91^{\circ}, 0.77^{\circ}$, or $0.49^{\circ}$. The duration of the target bars was one frame $(10 \mathrm{~ms})$.

Post bars The color of the post bars was white. Their luminance was $81.4 \mathrm{~cd} / \mathrm{m}^{2}$, and their duration was one frame $(10 \mathrm{~ms})$. Stimulusonset asynchrony (SOA) between target and post bars was always 10 frames $(100 \mathrm{~ms})$. Two post bars were presented. The post bars were centered at $2.22^{\circ} \mathrm{left}$ and right of and $2.22^{\circ}$ above the fixation dot. In the short right bar condition, the lengths of the right and left post bars were $0.7^{\circ}$ and $1.4^{\circ}$, respectively. In the short left bar condition, the lengths of the right and left post bars were $1.4^{\circ}$ and $0.7^{\circ}$, respectively.

\section{Procedure}

The experiments were conducted in a dark room. The observers sat at a distance of $50 \mathrm{~cm}$ from the CRT display and placed their head on a chinrest. The experiment began with their pressing the spacebar on the keyboard. After an interval of 25 frames $(250 \mathrm{~ms})$, a stimulus sequence (i.e., test bars for one frame [10 ms] followed by probe bars for one frame [10 ms] with the SOA of 10 frames [100 ms]) was presented. The task of the observers was to judge which of the right or left target bar was shorter than the other. The observers reported their judgments by pressing assigned keys. They were urged to judge bar lengths with 


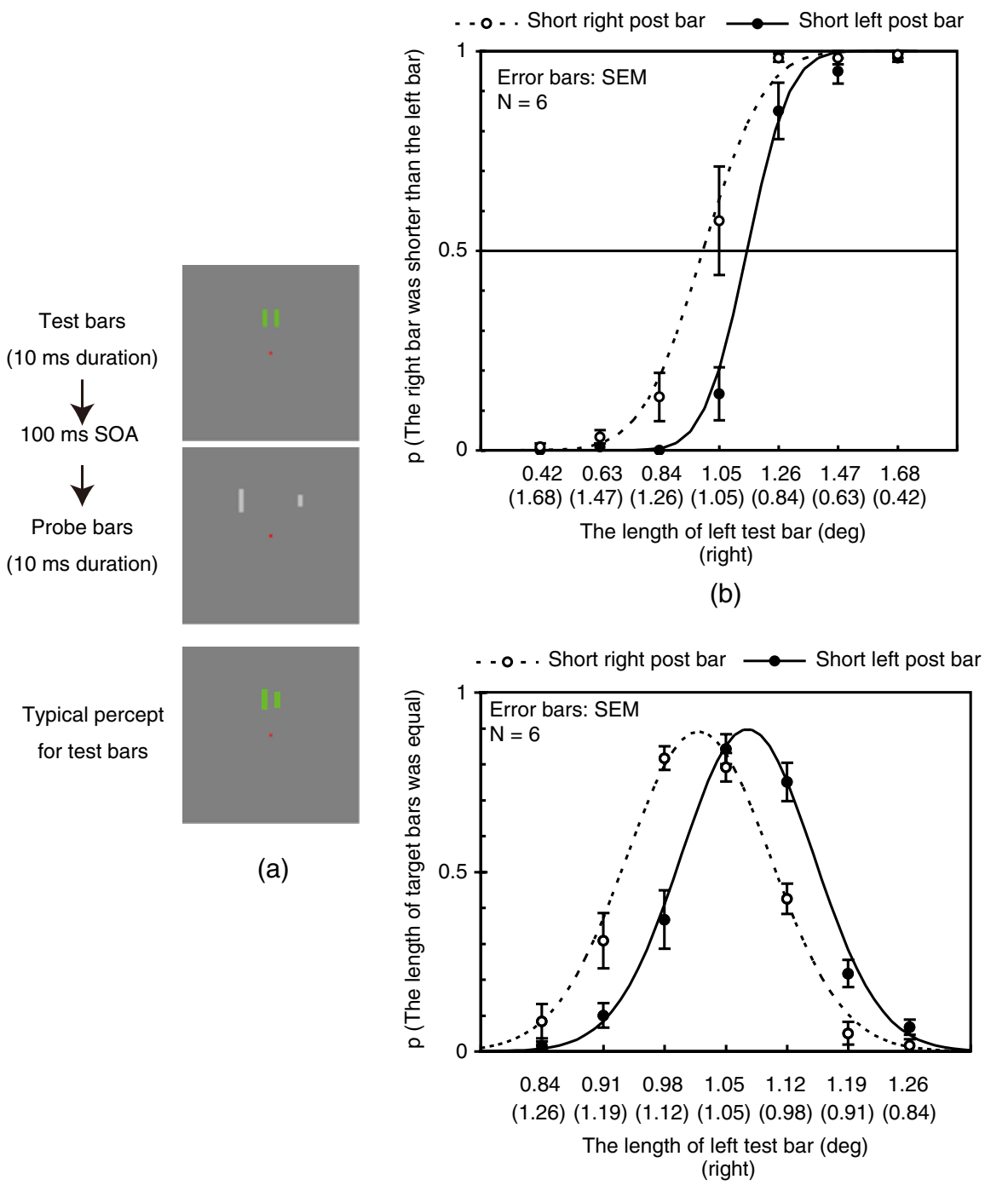

(d)

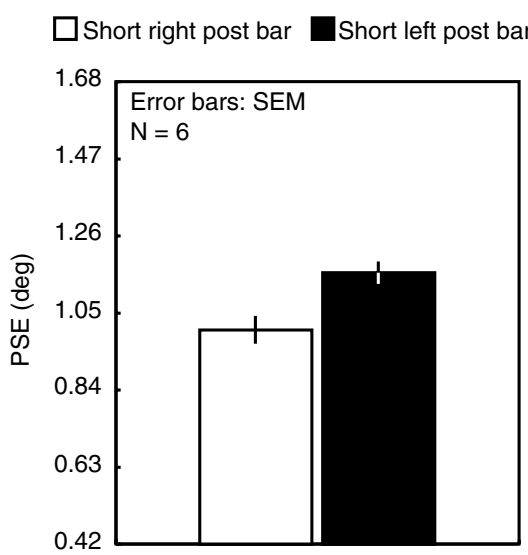

(c)

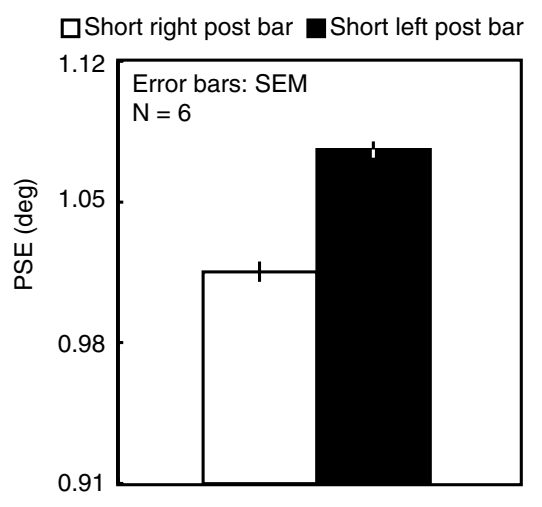

(e)
Fig. 2 aSchematic diagram of stimulus presentation in Experiment 1 and 2, representing the short right bar condition in which the right post bar is shorter than the left one. A typical percept for test bars is shown in the bottom panel. $(b-c)$ The Experiment 1 results: b Mean proportions of trials on which the right test bar was perceived to be shorter than the left test bar as a function of actual lengths of the left test bar. Error bars denote $\pm 1 S E M(N=6)$. $\mathbf{c}$ Group mean of the point of

the best guess even when it was difficult to judge. The next trial began $300 \mathrm{~ms}$ after the response. In Experiment 1, each observer received 280 trials of two post bar conditions (short left and short right bar conditions) $\times 7$ relative lengths conditions of test bars $\times 20$ replications. The order of trials was randomized across observers.

\section{Results and discussion}

For each condition, the proportion of trials on which the right test bar was reported to be shorter than the left test bar was calculated as a function of actual length of the left test subjective equality (PSE) for the left test bar to be reported as having alength equal to that of the right test bar. Error bars denote $\pm 1 \operatorname{SEM}(N=$ 6). Experiments 2 results: d Mean proportions of trials on which the observers reported test bars to have an equal length as a function of actual length of the left test bar. Error bars denote $\pm 1 \operatorname{SEM}(N=6)$. e Group mean of the PSE for the left test bar to be reported as having an equal length to the right test bar. Error bars denote $\pm 1 \operatorname{SEM}(N=6)$

bar. The resultant proportions averaged among observers are plotted in Fig. 2b. For each observer, a cumulative normal curve was fitted to the proportions as a function of actual lengths of the left bar in order to estimate points of subjective equality (PSEs) for the left test bar to be judged as being of equal length to the right test bar. The fitting was carried out by employing the Palamedes toolbox (Prins \& Kingdom, 2009). Goodness of fit was confirmed by overall low deviance and cumulative probability estimate (in all cases, $p>.05$ ). Estimated PSEs were averaged across the observers, and group mean data are shown in Fig. 3b for each post bar condition. A two-tailed $t$-test showed that the 


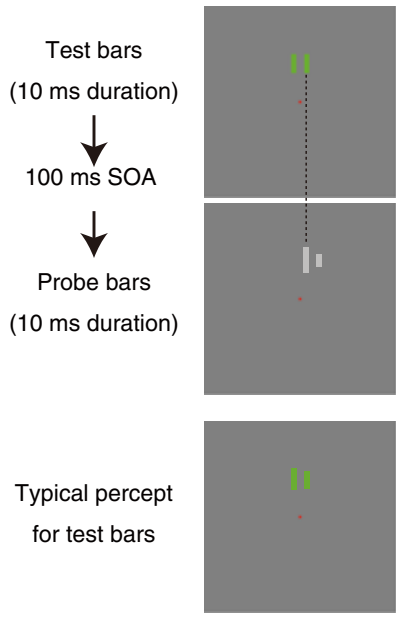

(a)

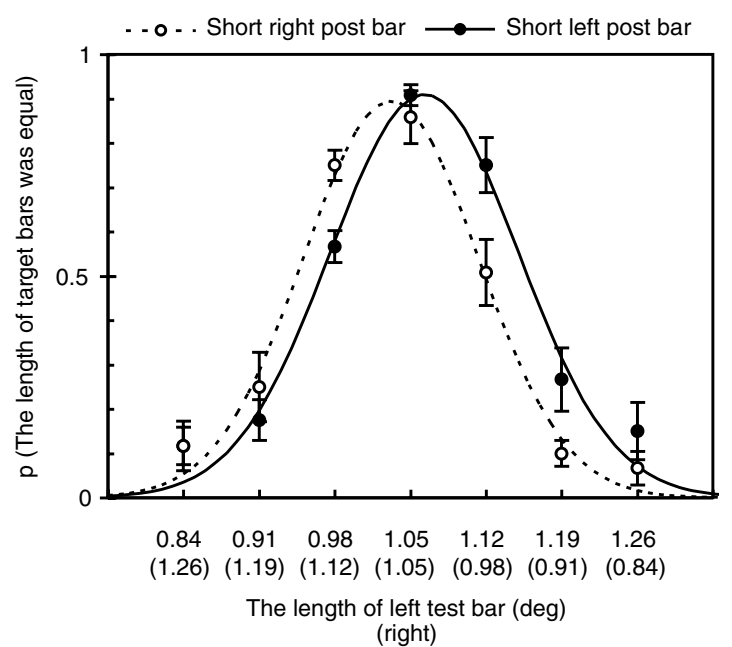

(b)

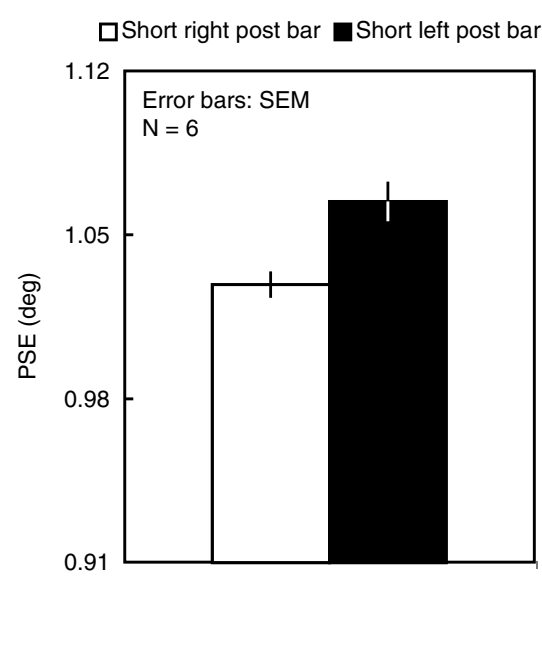

(c)
Fig. 3 aSchematic diagram of stimulus presentation in Experiment 3 , representing the short right bar condition in which the right post bar is shorter than the left one. A typical percept for test bars is shown in the bottom panel. b Mean proportions of trials on which the observers reported test bars to have an equal length as a function of actual length of the left test bar. Error bars denote \pm 1 SEM $(N=6)$. c Group mean of PSE for the left test bar to be reported as having an equal length to the right test bar. Error bars denote $\pm 1 \operatorname{SEM}(N=6)$

Anton-Erxleben, Abrams, \& Carrasco, 2010, for a counterexample). It was likely that the observers reported the length pattern of post bars as the one of target bars, leading to a nominal postdictive size modulation.

In this experiment, we alternatively employed an equality judgment task in which the observers were asked to report whether the lengths of target bars were equal or not. This task was free from the response bias of the kind described above, because on all trials, the lengths of the left and right post barswere different from each other by the same amount (i.e., $0.7^{\circ}$ ), and thus the observers could not base their judgments of the target bars on the post bars: If the observers had been relying their response on the length pattern of the post bars, they would have had to always report the length of the target bars to be different. We predicted that the postdictive size modulation would be observed if the modulation was a product of a perceptual process, not of a decisional process.

Method

Observers

Six observers (including the author, 1 person participating in Experiment 1, and 4 new people) participated in this experiment. All subjects had normal or corrected-to-normal visual acuity and gave written informed consent before the experiment. With the exception of the author, they were unaware of the purpose of the study. 


\section{Apparatus}

The apparatus was unchanged from Experiment 1.

\section{Stimuli}

The stimuli were identical to those used in Experiment 1, except for the following. The range of length differences between right and left target bars was narrowed, in comparison with the range used in Experiment 1. Their lengths were relatively changed on each trial; when the length of the left target bar was $0.84^{\circ}, 0.91^{\circ}, 0.98^{\circ}, 1.05^{\circ}, 1.12^{\circ}$, $1.19^{\circ}$, or $1.26^{\circ}$, the length of the right target bar was $1.26^{\circ}$, $1.19^{\circ}, 1.12^{\circ}, 1.05^{\circ}, 0.98^{\circ}, 0.91^{\circ}$, or $0.84^{\circ}$. As in Experiment 1 , there were two conditions involving the spatial pattern of the post bars. One was the short left post bar condition, in which when the length of the left post bar was $0.7^{\circ}$ and the length of the right post bar was $1.4^{\circ}$. The other was the short right post bar condition, in which when the length of the left post bar was $1.4^{\circ}$ and the length of the right post bar was $0.7^{\circ}$.

\section{Procedure}

Theprocedure was identical to that used in Experiment 1, except for the following. The task of the observers was to judge whether the lengths of the right and left target bars were equal or not. Each observer received 280 trials of two post bar conditions (short left and short right bar conditions) $\times 7$ relative lengths conditions of test bars $\times 20$ replications. The order of trials was randomized across observers.

\section{Results and discussion}

The proportion of trials on which the length of target bars was reported to be equal was calculated for each condition and is plotted in Fig. 2d. For the proportions, we conducted a twoway ANOVA with (1) length patterns of post bars (two levels: short left vs. right conditions) and (2) left test bar length (seven levels) as factors. The main effect of length patterns of post bars was significant, $F(1,5)=13.913, p<.02$. The main effect of left test bar length was also significant, $F(6,30)=$ $98.133, p<.0001$. The interaction of the two factors was also significant, $F(6,30)=22.628, p<.0001$. An important aspect of the posthoc test for the significant interaction was that there was a significant difference between short left and right post bar conditions when the left target bar length was $0.91^{\circ}$, $0.98^{\circ}, 1.12^{\circ}$, and $1.19^{\circ}(p<.005)$.

In addition, we fitted a Gaussian function to individual data of the proportions as a function of left target bar length in order to calculate PSE for test bar length. Averaged $r^{2}$ across observers was $.97(S D=.02)$, indicating that the fitting was well performed. The mean of the fitted function was considered as the PSE. The resultant PSEs, averaged across observers, are shown in Fig. 2e. For the PSE, we conducted a two-tailed $t$-test and acknowledged a significant difference between short left and right post bar conditions, $t(5)=8.70, p<.0004$.

The results demonstrated size modulation by postdictive motion signals even when the involvement of response bias was kept out, indicating that postdictive size modulation is a product of a perceptual process, not of a decisional process.

\section{Experiment 3}

This experiment was carried out to confirm whether the postdictive size modulation occurred in the nonretinotopic way (in other words, in an object-based manner). As was described in the introduction, visual features within an object are integrated across space and time (Boi et al., 2009; Öğmen et al., 2006; Otto et al., 2008). However, although these previous studies have focused on how visual features such as vernier offsets, motion, and orientation in a leading frame affectedthe appearance of these features in a trailing frame, they have not investigated whether the perceived length of an object could be modulated by the length of the object in a temporally separated frame.In the previous experiments in the present study, we demonstrated that the perceived length of the object in the first frame was strongly affected by the length of the object in the second frame when the motion correspondence betweenthe frames was determined in the retinotopic way. On the other hand, it was still unclear whether the postdictive size modulation existed when motion correspondence was determined in the nonretinotopic way.

To test this issue, we employed a new stimulus configuration that was similar to the Ternus-Pikler display, as shown in Fig. 3a (Ternus, 1926). In this stimulus configuration, the left and right test bars perceptually correspond to the left and right post bars when a temporal interval between frames is sufficiently long (i.e., >100 ms), despite the fact that the retinotopic location of the right test bar is identical to the one of the left post bar. By using this stimulus configuration, we tried to investigate how the length pattern of post bars would affectthe perceptual length of target bars when motion correspondence was determined in the nonretinotopic way.

Method

\section{Observers}

Six observers (including the author, 1 person who had participated in Experiments 1 and 2, and 4 new people who had not participated in any experiments) participated in this 
experiment. All subjects had normal or corrected-to-normal visual acuity and gave written informed consent before the experiment. With the exception of the author, they were unaware of the purpose of the study.

\section{Apparatus}

The apparatus wasunchanged from Experiment 1.

\section{Stimuli}

The stimuli were identical to those used in Experiments 2, except for the following. Spatial interval between post bars was $0.74^{\circ}$ (i.e., just identical to the spatial interval between test bars). The location of the left (or right) post bar was coincident with the one of the right (left) test bar (see Fig. 3a). Either of the two locations was randomly selected on each trial. In the short right bar condition, the lengths of the right and left post bars were $0.7^{\circ}$ and $1.4^{\circ}$, respectively. In the short left bar condition, the lengths of the right and left post bars were $1.4^{\circ}$ and $0.7^{\circ}$, respectively. SOA between target bars and post bars was 15 frames $(150 \mathrm{~ms})$. Instead of the 100-ms SOA used in Experiments 2, an SOA of $150 \mathrm{~ms}$ was employed because, in preliminary experiments, some observers could not perform the task, due to the low visibility of target bars.

\section{Procedure}

Theprocedure was identical to that in Experiments 2, except for the following. In Experiment 3, we conducted a preliminary observation, consisting of 10 trials, ahead of the main experiment in order to briefly confirm whether the observers actually would perceive a group motion in the Ternus-Pikler displaylike stimuli we employed. The observers were presented stimuli that were just identical to those used in the main experiment and were asked to report whether they perceived element motion with a motion correspondence between the left (and right) target bar and the right (and left) post bar or group motion with a motion correspondence between the left (and right) target bar and the left (and right) post bars. As a result, all of them exclusively reported group motion in 10 of 10 trials. Thus, we concluded that the stimuli we employed were suitable for testing the contribution of nonretinotopic processing in the postdictive modulation of size perception in apparent motion. In the main experiment, the task of the observers was to judge whether the lengths of the right and left target bars were equal or not. Each observer received 280 trials of two post bar conditions (short left and short right bar conditions) $\times 7$ relative length conditions of test bars $\times 20$ replications. The order of trials was randomized across observers.
Results and discussion

The proportion of trials on which the length of target bars was reported to be equal was calculated for each condition and is plotted in Fig. 3b. For the proportions, we conducted a twoway ANOVA with (1) length patterns of post bar (two levels: short left vs. right conditions) and (2) left test bar length (seven levels) as factors. The main effect of length patterns of the post bar was significant, $F(1,5)=7.81, p<.04$. The main effect of left test bar length was also significant, $F(6,30)=59.470, p<.0001$. The interaction of the two factors was also significant, $F(6,30)=6.00, p<.0003$. An important aspect of the posthoc test for the significant interaction was that there was a significant difference between short left and right post bar conditions when the left target bar length was $0.98^{\circ}, 1.12^{\circ}$, and $1.19^{\circ} \operatorname{deg}(p<.005)$.

As in Experiments 2, we calculated PSEs for test bar length by fitting a Guassian curve to the proportions as a function of the left test bar length. Averaged $r^{2}$ across observers was .93 $(S D=.09)$, again indicating that the fitting was well performed. The mean of the fitted function was considered as a PSE. The resultant PSEs, averaged across observers, are shown in Fig. 3c. On the PSE, we conducted a two-tailed $t$-test and acknowledged a significant difference between short left and right post bar conditions, $t(5)=3.04, p<.03$.

The results indicate that postdictive size modulation occurred even when the postdictive motion signals were produced on the basis of nonretinotopic information, suggesting that postdictive size modulation is conducted in an object-based manner that is intimately related to nonretinotopic information. That is, the visual system seems to determine motion correspondence for every perceptual object (i.e., a bar, in our case), and depending on the motion correspondence, the visual system postdictively estimates the past size of the object. These issues will again be touched upon in the General Discussion section. Moreover, as in Experiments 2, postdictive size modulation occurred in the equality judgment task, again supporting that not a decisional process, but a perceptual process is involved in size modulation by postdictive motion signals.

\section{General discussion}

This study demonstrated that motion correspondence on the basis of retinotopic and nonretinotopic information was linked to postdictive size modulation in a two-stroke apparent motion. Moreover, the postdictive size modulation did not stem from a bias to respond to the length pattern of the post bars.

The present study supports the idea that the content of visual awareness for earlier input is overwritten by later input (Dennett \& Kinsbourne, 1992). Interestingly, the 
results of Experiment 3 suggested that the overwriting of conscious contents occurs on the basis of object processing of nonretinotopic properties. One type of object processing reported previously, which seems to be related to this overwriting, is object updating (Lleras \& Moore, 2003; Moore \& Enns, 2004). Here, a brief description of object updating and the relevant literature are presented. To construct a stable mental world from fluctuating and ambiguous inputs from the external world, the visual system must determine whether the current input should be matched to an existing object or should be regarded as a newly formed object. To do so, the visual system tries to update the earlier representation of the object by comparing it with the later input information. The theory of object updating assumes that when the later input information at a different location is perceptually relevant to the earlier representation, the earlier representation of the object is suppressed, and the representation of the object is updated on the basis of the later input information. On the other hand, when the later input information at a different location is perceptually irrelevant to the earlier representation, the earlier representation of the object is not disrupted, and a new object is perceptually formed with the later input information. This assumption has been empirically confirmed in studies of the flash-lag effect (Moore \& Enns, 2004), object substitution masking (Lleras \& Moore, 2003), and the judgment of visible persistence of a flash in an apparent motion trajectory (Moore, Mordkoff, \& Enns, 2007).

We suppose that object updating is intimately related to postdictive size modulation in two-stroke apparent motion. As was described in the introduction, when a triangle flash is followed by a square flash, observers report the apparent motion trajectory within which the flash shape gradually changes from a triangle to a square (Kolers \& von Grünau, 1976). Kolers and Pomerantz (1971) called this kind of shape change along the apparent motion trajectory "plastic deformation." Lleras and Moore (2003) reported that plastic deformation is a visual illusion reflecting the objectupdating process. That is, in a two-stroke apparent motion, an earlier flash is subject to overwriting by a later flash as long as object representation of the earlier flash is linked to the later flash. Temporal changes in object representation during object updating may be the source of the plastic deformation. Actually, a previous study showed that common-onset masking, which is caused by object updating, alters the appearance of an earlier target (Kahan \& Enns, 2010).

In Experiment 1 of the present study, when motion correspondence was assigned between test bars with equal lengths and a longer or shorter post bar, the observers reported the test bars to be longer or shorter, respectively. We suggest that the visual system might sample a later point in changing representation of a flash during object updating, and thus the point may be reported as the length of the actually presented test bars, leading to postdictive size modulation toward the length of probe bars. Alternatively, the representation of a flash during object updating might be averaged, leading to postdictive size distortion of the test bars toward the size of the post bars. In another line of research, a psychophysical mechanism that averages spatial representation across time has been proposed (Krekelberg \& Lappe, 1999, 2000; Roulston, Self, \& Zeki, 2006), and a similar mechanism might be related to the postdictive size modulation discussed here.

It was also demonstrated that postdictive size modulation occurred in accordance with motion correspondence based on nonretinotopic information. To our knowledge, this is the first study to show that motion correspondence based on nonretinotopic information postdictively alters the appearance of an earlier representation. We suppose that object updating is also involved in postdictive modulation on the basis of motion correspondence with nonretinotopic information. A previous study showed that visual features within an object were retained across a two-stroke apparent motion when motion correspondence was established with nonretinotopic information (Boi et al., 2009). That is, there is a possibility that visual features within an object are stored and integrated across space and time. Spatiotemporal integration of visual features within an object has been investigated by using the phenomenon called feature attribution, in which an earlier representation alters the appearance of a later frame in a predictive way (Kawabe, 2008; Öğmen et al., 2006; Otto et al., 2008). The results of the present study suggest that the integration of visual features across space and time occurs not only in a predictive manner, but also in a postdictive manner. In future studies, we will specify the precise relationships between predictive and postdictive visual feature integration across space and time.

In addition to object updating, motion deblurring and its relevant outcomes might play a key role in postdictive size modulation. The duration of visible persistence of a flash is approximately 100-120 ms (Haber \& Standing, 1970). Given the duration of visible persistence, a moving object should produce retinal smears along its trajectory. However, in our daily experience, this is not the case. Previous studies have demonstrated that the visual system has a function for deblurring retinal smears along a motion trajectory (Burr, 1980; Chen, Bedell, \& Öğmen, 1995). This kind of deblurring mechanism is related to spatiotemporal summation of successive visual frames (Burr, 1984). Moreover, spatiotemporal summation (or averaging) of visual features occurs along a motion trajectory of a visual object (Cavanagh, Holcombe, \& Chou, 2008; Nishida, Watanabe, Kuriki, \& Tokimoto, 2007). In the present study, due to 
spatiotemporal summation (or averaging) based on motion deblurring or a similar visual function, the size of a bar in the leading frame is averaged with the size of a bar in the trailing frame along a motion trajectory, leading to postdictive size modulation of object size. It is known that motion deblurring works in an object-based manner (Moore et al., 2007), and thus it is also possible that a similar kind of summation (or averaging) occurred in our stimulus configuration, causing nonretinotopic motion correspondence. It would seem to be hard, but worth the challenge, to disentangle the effect of object updating from the effect of motion deblurring on postdictive size modulation in future studies.

It is unclear whether the present results involved motioninduced position shifts of a flash. Eagleman and Sejnowski (2007) suggested that motion-induced position shift of a flash plays a key role in the flash-drag, flash-jump, and Fröhlich effects. Previous studies have suggested that motion-induced position shift of a flash occurs in the direction of attention shift (Shim \& Cavanagh, 2004, 2005; Watanabe, 2005), and the results of Eagleman and Sejnowski were consistent with the evidence. However, the results of the present study demonstrated postdictive size modulation occurring in the orthogonal direction (i.e., vertical dimension) to possible attention shift (i.e., horizontal dimension). Thus, it is premature to conclude that a motion-induced position shift of a flash was involved in the postdictive size modulation in this study. Moreover, no previous studies have addressed whether the motion-induced position shift of a flash occurs in an object-based manner. Further studies are warranted to clarify what kinds of interaction of perceptual processing for object formation, apparent motion, and localization underlie postdictive size modulation.

Acknowledgment The authors would like to thank Michael $\mathrm{H}$. Herzog, James T. Enns, and Alex O. Holcombe for valuable comments and suggestion on an earlier version of the manuscript. The authors also thank the observers who participated in the present experiments. This work was supported by the SSP project at Kyushu University.

\section{References}

Anton-Erxleben, K., Abrams, J., \& Carrasco, M. (2010). Evaluating comparative and equality judgments in contrast perception: Attention alters appearance. Journal of Vision, 10(11, Art. 6), $1-22$.

Boi, M., Öğmen, H., Krummenacher, J., Otto, T. U., \& Herzog, M. H. (2009). A (fascinating) litmus test for human retino- vs. nonretinotopic processing. Journal of Vision, 9(13, Article 5), 1-11. Retrieved from http://journalofvision.org/9/13/5/, doi:10.1167/9.13.5

Brainard, D. H. (1997). The psychophysics toolbox. Spatial Vision, $10,433-436$.

Breitmeyer, B. G., Herzog, M. H., \& Ogmen, H. (2008). Motion, not masking, provides the medium for feature attribution. Psychological Science, 19, 823-829.

Burr, D. C. (1980). Motion smear. Nature, 284, 164-165.
Burr, D. C. (1984). Summation of target and mask metacontrast stimuli. Perception, 13, 183-192.

Cai, R. H., \& Schlag, J. (2001). Asynchronous feature binding and the flash-lag illusion. Investigative Ophthalmology \& Visual Science, 42, S711.

Carbone, E., \& Ansorge, U. (2008). Investigating the contribution of metacontrast to Fröhlich effect for size. Acta Psychologica, 128, $361-367$.

Cavanagh, P., Holcombe, A. O., \& Chou, W. (2008). Mobile computation: Spatiotemporal integration of the properties of objects in motion. Journal of Vision, 8(12, Art. 1), 1-23.

Chen, S., Bedell, H. E., \& Öğmen, H. (1995). A target in real motion appears blurred in the absence of other proximal moving targets. Vision Research, 35, 2315-2328.

Choi, H., \& Scholl, B. J. (2006). Perceiving causality after the fact: Postdiction in the temporal dynamics of causal perception. Perception, 35, 385-399.

Dennett, D. C., \& Kinsbourne, M. (1992). Time and the observer: The where and when of consciousness in the brain. The Behavioral and Brain Sciences, 15, 183-247.

Eagleman, D. M., \& Sejnowski, T. J. (2007). Motion signals bias position judgments: A unified explanation for the flash-lag, flashdrag, flash-jump and Frohlich effects. Journal of Vision, 7(4, Art. 3), 1-12. Retrieved from http://journalofvision.org/7/4/3

Freyd, J. J. (1987). Dynamic mental representations. Psychological Review, 94, 427-438.

Fröhlich, F. W. (1923). Über die messung der empfindungszeit. Zeitschrift für Sinnesphysiologie, 54, 58-78.

Haber, R. N., \& Standing, L. (1970). Direct estimates of the apparent duration of a flash. Canadian Journal of Psychology, 24, 216-229.

Kahan, T. A., \& Enns, J. T. (2010). Object trimming: When masking dots alter rather than replace target representation. Journal of Experimental Psychology. Human Perception and Performance, $36,88-102$.

Kawabe, T. (2008). Spatiotemporal feature attribution for the perception of visual size. Journal of Vision, 8(8, Art. 7), 1-9. Retrieved from http://journalofvision.org/8/8/7/, doi:10.1167/8.8.7

Kerzel, D. (2003). Mental extrapolation of target position is strongest with weak motion signals and motor responses. Vision Research, 43, 2623-2635.

Kerzel, D., Zarian, L., Gauch, A., \& Buetti, S. (2010). Large effects of peripheral cues on appearance correlate with low precision. Journal of Vision, 10(11, Art. 26), 1-14. Retrieved from http:// www.journalofvision.org/content/10/11/26, doi:10.1167/10.11.26

Kirschfeld, K., \& Kammer, T. (1999). The Fröhlich effect: A consequence of the interaction of visual focal attention and metacontrast. Vision Research, 39, 3702-3709.

Kolers, P. A., \& Pomerantz, J. R. (1971). Figural change in apparent motion. Journal of Experimental Psychology, 87, 99-108.

Kolers, P. A., \& von Grünau, M. (1975). Visual construction of color is digital. Science, 187, 757-759.

Kolers, P. A., \& von Grünau, M. (1976). Shape and color in apparent motion. Vision Research, 16, 329-335.

Krekelberg, B., \& Lappe, M. (1999). Temporal recruitment along the trajectory of moving objects and the perception of position. Vision Research, 39, 2669-2679.

Krekelberg, B., \& Lappe, M. (2000). A model of the perceived relative positions of moving objects based upon a slow averaging process. Vision Research, 40, 201-215.

Lleras, A., \& Moore, C. M. (2003). When the target becomes the mask: Using apparent motion to isolate object-level component of object substitution masking. Journal of Experimental Psychology. Human Perception and Performance, 29, 106-120.

Maus, G. W., \& Nijhawan, R. (2006). Forward displacements of fading objects in motion: The role of transient signals in perceiving position. Vision Research, 46, 4375-4381. 
Maus, G. W., \& Nijhawan, R. (2008). Motion extrapolation into the blind spot. Psychological Science, 19, 1087-1091.

Moore, C. M., \& Enns, J. T. (2004). Object-updating and the flash-lag effect. Psychological Science, 15, 866-871.

Moore, C. M., Mordkoff, J. T., \& Enns, J. T. (2007). The path of least persistence: Object status mediates visual updating. Vision Research, 47, 1624-1630.

Nijhawan, R. (1994). Motion extrapolation in catching. Nature, 370, $256-257$.

Nijhawan, R. (2002). Neural delays, visual motion, and the flash-lag effect. Trends in Cognitive Sciences, 6, 387-393.

Nijhawan, R. (2008). Visual prediction: Psychophysics and neurophysiology of compensation for time delays. The Behavioral and Brain Sciences, 31, 179-239.

Nishida, S., Watanabe, J., Kuriki, I., \& Tokimoto, T. (2007). Human visual system integrates color signals along a motion trajectory. Current Biology, 17, 366-372.

Öğmen, H., Otto, T., \& Herzog, M. H. (2006). Perceptual grouping induces non-retinotopic feature attribution in human vision. Vision Research, 46, 3234-3242.

Otto, T. U., Öğmen, H., \& Herzog, M. H. (2008). Assessing the microstructure of motion correspondences with non-retinotopic feature attribution. Journal of Vision, 8(7, Art. 16), 1-15. Retrieved from http://journalofvision. org/8/7/16/, doi:10.1167/ 8.7.16

Pelli, D. G. (1997). The VideoToolbox software for visual psychophysics: Transforming numbers into movies. Spatial Vision, 10, 437-442.

Prins, N., \& Kingdom, F. A. A. (2009). Palmedes: Matlab routines for analyzing psychophysical data. Retrieved from http:// www. palamedestoolbox.org/
Prinzmetal, W., Long, V., \& Leonhardt, J. (2008). Involuntary attention and brightness contrast. Perception \& Psychophysics, $70,1139-1150$.

Roulston, B. W., Self, M. W., \& Zeki, S. (2006). Perceptual compression of space through position integration. Proceedings of Royal Society of London B, 273, 2507-2512.

Schneider, K. A., \& Komlos, M. (2008). Attention biases decisions but does not alter appearance. Journal of Vision, 8(15, Art. 3), 110. Retrieved from http://www.journalofvision.org/ content/8/15/ 3, doi:10.1167/8.15.3

Shi, Z., \& Nijhawan, R. (2008). Behavioral significant of motion direction causes anisotropic flash-lag, flash-drag, flash-repulsion, and movement-mislocalization effects. Journal of Vision, 8(7, Art. 24), 1-14. Retrieved from http://journalofvision.org/8/7/24/, doi: $10.1167 / 8.7 .24$

Shim, W. M., \& Cavanagh, P. (2004). The motion-induced position shift depends on the perceived direction of bistable quartet motion. Vision Research, 44, 2393-2401.

Shim, W. M., \& Cavanagh, P. (2005). Attentive tracking shifts the perceived location of a nearby flash. Vision Research, 45, 32533261.

Shim, W. M., \& Cavanagh, P. (2006). Bi-directional illusory position shifts toward the end point of apparent motion. Vision Research, 46, 3214-3222.

Ternus, J. (1926). Experimentelle untersuchungen über phänomenale identität. Psychological Research, 7, 81-136.

Watanabe, K. (2005). The motion-induced position shift depends on the visual awareness of motion. Vision Research, 45, 2580-2586.

Whitney, D., \& Cavanagh, P. (2000). Motion distorts visual space: Shifting the perceived position of remote stationary objects. Nature Neuroscience, 3, 954-959. 